SAMUEL LAIR

Université d'Angers

\title{
OCTAVE MIRBEAU ET LES CLIVAGES DU MOI
}

\begin{abstract}
Lair Samuel, Octave Mirbeau et les clivages du moi [Octave Mirbeau's rifts]. Studia Romanica Posnaniensia, Adam Mickiewicz University Press, Poznań, vol. XXXII : 2005, pp. 123-142. ISBN 83-232-1465-4, ISSN 0137-2475.
\end{abstract}

Mirbeau's inner world, with its hidden treasures, its disorder, jts constructive incoherence, creates a literature in its own image, violent, disparate and generous. His entire work is part of his contemporary Viennese master's one. Subconscious deliberate mistakes, sublimations, perversions, original scenes, neuroses... Everything leads the reader to integrate the biographical element into the very act of creation.

The regressive attitude of his earlier novels which translates into a desire for fusion with Nature lurns in the 1890 s into a will of self-construction eventually opening onto a confident vitality and an enthusiastic belief in modernity.

But this decadent literature, no more than a naturalistic one, should not be reduced to a psychoanalytic analysis. Should they exist, these elements are subjected to a literary transmutation which urges the reader to conceive his own personal myth rather than sense a neurotic divide. Torn between an intellectual demand and a spiritual expectation, it seems nonetheless that Nature, the element of inspiration for the author, lets us feel a prospective deep division.

Un aspect de la modernité de Mirbeau bien souvent est laissé dans l'ombre : l'intuition aiguë d'une activité psychique obscure, débordante, qui régit activement le cours de la vie individuelle comme sociale, annonce l'orientation des découvertes et des travaux menés par la psychanalyse. En de nombreux cas, l'imaginaire de Mirbeau se repaît d'images érotiques, de représentations sensuelles que rien ne semble justifier - ni les nécessités de l'intrigue qui n'en tire qu'un profit relatif, sauf à la rigueur pour en nourrir les portraits de personnages, ni l'aspiration vers la transcendance du Bien ou du Beau héritée de la sensibilité romantique. Bien souvent, il semble que l'amour n'ait pour autre fin que lui-même ou la mort, dans des cuvres qui à ce titre portent en germes les centres d'intérêt que se fixera plus tard l'initiateur de la psychanalyse. En 1924, Maxime Revon ne dit pas autre chose :

Notons que si Mirbeau écrivait maintenant son Abbé Jules, des critiques avertis ne manqueraicnt pas d'y remarquer une influence des théories de M. Sigismond Freud [sic], du 
moins la leçon des œuvres de Mirbeau peut-elle servir d'illustration aux démonstrations du professeur viennois'.

Du reste, le "petit Rousseau " et "le terrible affronteur " ${ }^{2}$ qu'il a lui-même été, l'être de la nature, le Prométhée anarchiste, pour lesquels il plaide tout au long de sa vie, justifieraient à eux seuls qu'on aille chercher du côté de la psychanalyse quelques lumières sur les fantasmes, les mobiles secrets et les arcanes profonds de cet écrivain individualiste, même en l'absence de signes romanesques patents. En l'occurrence ils existent en grand nombre, et de manière très significative. Ces traces symptomatiques engagent à en dresser le catalogue.

\section{VIVACITÉ DES REPRÉSENTATIONS DE LA CULPABILITÉ}

Force est de constater qu'au milieu des vices et des formes innombrables de perversité dont l'œuvre offre une éloquente galerie, les manifestations de culpabilité font figure de malaise bénin; et pourtant les attitudes des personnages qui en portent les traces sont lourdes de conséquences. Ce que l'on peut en retenir de prime abord, c'est l'aspect de mécanique tournant à vide que prend ce sentiment de faute angoissant et invalidant : rares sont les accès de culpabilité « légitime », justifiée par une conduite réellement fautive et responsable d'un préjudice à l'égard d'autrui. Les personnages de Mirbeau culpabilisent plus qu'ils ne sont coupables.

L'une des uniques représentations d'une faute caractérisée et suscitant chez son auteur le désir de repentance figure dans la première tentative d'écriture de Mirbeau encore adolescent, Le Meurtre des enfants d'Édouard (1863); l'odieux Tyrrène, auteur du meurtre des deux enfants d'Édouard IV commandité par leur oncle le duc de Gloucester, se montre en fin de récit capable de remords et d'expiation. Le sentiment de culpabilité coïncide avec la morale, et Mirbeau donne là à lire une fin globalement optimiste. Il en va autrement par la suite, où dans un mouvement spontané autant qu'infondé, les personnages connaissent les affres d'une faute qu'ils n'ont pas commise, au mépris de toute raison, et plus parfois, à rebours de toute logique morale ou psychologique. Les victimes constituent chez Mirbeau la principale communauté de «repentants ». Après le viol dont il a été victime, Sébastien est dévoré de remords cuisants et ne parvient à se soustraire aux idées de culpabilité et de péché, quand bien même l'intégralité du crime relève de la responsabilité de de Kern; le dégoût et la honte qui président à son premier contact avec Marguerite paraissent s'ancrer dans la douleur de cette expérience relayée par un enseignement religieux qui exalte la repentance.

De manière générale, les relations amoureuses offrent un terrain propre à aiguillonner chez les personnages le sentiment de la faute. Julia, la petite amie du

\footnotetext{
'Octave Mirbeau, son æuvre, La Nouvelle Revue critique, 1924, p. 16.

${ }^{2}$ Joumal, Goncourt, Robert Laffont, Collection Bouquins, II, p. 314.
} 
narrateur de Dans le ciel, exprime après leur première étreinte ce désarroi imprégné de remords : “C'est mal... C'est mal...»" ${ }^{3}$. En outre, à l'instar de la scène de l'assassinat des enfants d'Édouard, on note que le point culminant de la détresse, mais aussi le déclenchement de la crise de culpabilité, est atteint au moment de l'imploration pathétique et désespérée de la mère : Julia fait entendre de «petits vagissements ", avant de "balbuti[er] : - Maman !... Maman !... », cédant au même réflexe de défense que les deux petites victimes royales : «Ma mère ! Ma mère ! ». Les expressions de cette volonté de repentir décrivent une gamme variée dont la force dramatique est fonction de l'intrigue : le processus de sublimation esthétique à l'œuvre chez le peintre Lucien le conduit à mutiler sauvagement cet outil imparfait et indocile qu'est sa main ; la pieuse et superstitieuse Madame Mintié déplace l'effet de ses angoisses et de ses troubles sur le terrain de la religion : la séparation volontaire de son fils figure le tribut qu'elle s'impose de payer, en lointain écho de ce protagoniste d'une nouvelle qui, devançant le malheur inexorable, s'empresse d'étrangler de ses propres mains les deux oiseaux qui lui tenaient lieu de compagnons. Indéniablement, toutes ces illustrations témoignent plus du sentiment multiforme de la culpabilité chrétienne, qui recherche le châtiment comme une voie de salut providentielle, que d'une volonté d'autopunition laïcisée, celle que Freud affranchit de la notion de manquement moral. Comme leur auteur, les personnages possèdent une conscience aiguë de l'imprégnation d'une culture religieuse. Témoins de la vivacité du souhait d'expiation, certaines initiatives de réparation passent par le souhait récurrent d'évangéliser les âmes dont on imagine qu'elles sont dans l'erreur ou le péché. Simples velléités bien souvent, mais qui l'espace d'un instant, laissent entrevoir la possibilité de solder une dette morale pesante. Jean Mintié ${ }^{4}$ nourrit de façon éphémère le désir «d'entrer dans les bouges afin d'évangéliser les malheureuses créatures qui croupissent dans le vice, sans une bonne parole " ${ }^{5}$; l'abbé Jules fixe d'identiques objets à son désir de contrition, qui culmine lors de crises paroxystiques alternant sans logique avec des périodes d'exaltation et d'excitation charnelle ; Sébastien Roch formule de la façon la plus claire le sentiment du poids inexorable de l'éducation religieuse sur sa sensibilité désormais hantée par la faute :

Je ne puis, si confuse qu'clle soit cncore, me faire une conception morale de l'univers, affranchie de toutes les barbaries religieuses et sociales inculquécs au collège [...] Je ne puis même imaginer une forme d'art libre, sans me demander en même temps : «N'cst-ce pas un péché ? ${ }^{6}$

${ }^{3}$ Dans le ciel, L'Échoppe, Caen, 1989, p. 115.

${ }^{4}$ La conception chrétienne de la faute et de son rachat ne se lit nulle part ailleurs plus ostensiblement que dans Le Calvaire: "Des fautes conme les miennes, que je ne tente pas d'expliquer par l'influence des fatalitćs ataviques et par les pernicieux effets d'une éducation si contraire à ma nature, ont besoin d'unc expiation terrible [...] J'espère, quoique je ne croie plus en Dieu, [...] j'espère que j'aurai ma part des pitićs et des pardons chrétiens », p. 166.

${ }^{5}$ Le Calvaire, Mercure de France, 1991, p. 311.

${ }^{6}$ Sébastien Roch, Mercure de France, 1991, p. 985. 
Enfin comment ne pas mentionner ce roman mort-né, au titre évocateur, $\mathrm{La}$ Rédemption, qui devait faire suite au Calvaire et montrer le rachat de l'âme du héros au contact de la nature salvatrice et apaisante, roman que Mirbeau annonce à renfort de lettres et de publicité, à ses amis et aux directeurs de journaux, en prétendant qu'il est déjà comme achevé... et qu'il ne lui reste qu'à l'écrire ? ${ }^{7}$ Comment, sinon en soulignant que cet avortement illustre "l'acte manqué » par excellence, ô combien significatif de la difficulté de donner corps à certaines représentations essentielles?

\section{SUBLIMATION DES CAPACITÉS DE SENSIBILITÉ ESTHÉTIQUE SOUS LE TRAVAIL DU DÉSIR}

En effet, l'expérience de la sexualité ou du crime (puisque aussi bien les personnages eux-mêmes n'ignorent rien de l'origine unique de ce qui n'est que deux formes d'une même réalité) débouche ponctuellement sur l'ouverture d'un horizon jusqu'ici inconnu des personnages, qui outrepasse les bornes étroites de la simple jouissance amoureuse. Le comble de l'exaltation sensuelle s'accompagne de la sensation de réintégrer ce que l'homme possède de plus profond et de plus intime, de recouvrer ce dont le spolient les appauvrissantes structures sociales. La Beauté pénètre enfin la conscience de l'homme à la faveur de cette montée du désir qui intéresse indissociablement les domaines du corps et ceux de l'éthique et de l'esthétique $^{8}$.

De la lépreuse Annie qui à l'article de la mort contente sa passion des perles « une sorte de joie physique, de spasme charnel »" - et se plaît « à les toucher, à les caresser, à les baiser », à la belle Clara qui se repaît de la splendeur plastique du corps des suppliciés ${ }^{10}$, l'exaltation sensuelle mène à son apex une disponibilité totale à l'art, à moins que ce ne soit l'inverse. Reste qu'il est inconcevable pour l'initiatrice du jardin de cantonner l'une des jouissances dans un espace imperméable à l'autre. Un seul et mêtne désir aiguillonne l'âme vers les hauteurs inaccessibles à la volonté quotidienne.

On voit donc que malgré l'indissociable intrication de l'amour et du crime selon un processus qui veut que l'un soit la fin poursuivie par l'autre, la perspicacité des théories freudiennes échouerait à rendre compte de la sorte de transcendance des pulsions sexuelles, rendues contingentes par ce mouvement de dépassement. Au point que c'est parfois la volonté consciente des personnages qui paraît prendre le

'Il révèle à Paul Bourget qu'il a « de bien belles choses à dire ", et qu'il «n'[a] même plus qu’à les dire ", cité dans P. Michel et J.-F. Nivet, Octave Mirbeau, l'imprécateur an caur fidèle, Séguier, 1991, p. 306.

\footnotetext{
${ }^{\mathrm{s}}$ Lire par exemple Le Jardin des supplices, Folio, Gallimard, p. 162.

${ }^{9}$ Ibidem, p. 145.

${ }^{10}$ Ibidem, p. 166.
} 
relais ou anticiper sur cette sublimation de la pulsion de mort, la ligne de démarcation entre littérature et psychanalyse apparaît sensiblement dans ces scènes où des motifs relevant d'un élitisme esthétique ou de la revendication d'une exigence de hauteur morale poussent les protagonistes à rêver ou à donner libre cours à leurs aspirations au crime ${ }^{11}$. En marge des accomplissements sexuels ou meurtriers morbides, les plus éloquentes rencontres de cette sublimation du désir par la beauté ne sont-elles pas, plutôt que le fait d'hommages de l'amour à l'art, de l'art à l'amour qu'elles dotent de sens, qu'elles lestent d'une signification? L'ardeur génésique se confond clairement avec la sensibilité à la sensualité des formes, à la perfection des équilibres, au caractère irréprochable des contours et des volumes ${ }^{12}$.

\section{LA SCĖNE ORIGINAIRE. \\ TENTATIVE D’ÉCLAIRAGE DE CERTAINES RÉCURRENCES ROMANESQUES}

Intuition que se joue là quelque chose de déterminant dans l'esprit de l'enfant, volonté goguenarde de choquer en heurtant non seulement les lecteurs puritains mais les sensibilités attachées à une conception désexualisée du rapport parental, ou pourquoi pas récit d'un épisode ancré dans le terreau de l'expérience personnelle ? Les deux occurrences de la scène originaire qui figurent dans l'œuvre - dans $L^{\prime}$ 'Abbé Jules ${ }^{13}$, où elle est surprise par un jeune enfant qui s'en trouve violemment choqué, et dans "Les Mémoires de mon ami»" ${ }^{14}$ ne laissent pas d'éveiller la curiosité du chercheur et de susciter de multiples interrogations. Quel sens donner à cette incroyable préfiguration de ce qui formera, quelque vingt-cinq ans plus tard, le fondement même de l'étude décisive menée par Freud, à partir de 1910, sur le cas de «l'homme aux loups »? De manière un peu marginale, ajoutons que l'exploitation polémique de la sexualité, visant à démystifier l'amour, formait l'un des motifs de la répugnance de Freud à l'endroit de l'œuvre romanesque de Mirbeau, tandis que celle de Maupassant ne lui inspirait qu'éloges et admiration ${ }^{15}$.

\footnotetext{
${ }^{11}$ Quelques illustrations : Dans le ciel, p. 49 ; Le Jardin des supplices, p. 56.

${ }^{12}$ Particulièrement représentative, la chronique Aristide Maillol, La Revue, $1^{\text {er }}$ avril 1905, reprise dans Combats Esthétiques, II, pp. 382 et 388.

${ }^{13}$ L'Abbé Jules, Mercure de France, pp. 580-581.

${ }^{14}$ «Une nuit, je fus réveillé en sursaut... [...] Que s'était-il passé ?... Qu'avais-je vu ?... Qu'avaisje entendu ?... Je ne saurais le dire exactement ; ce que je sais, c'est que, sous l'impression de quelque chose d'anormal qui m'effraya, un craquement du lit, des voix rauques, des voix étouffées qui venaient du lit, des voix qui ressemblaient à des gémissements et à des râles... Je me dressais, soudain [...] je me mis à crier : ... Papa qui bat maman ! ... Papa qui tue maman! », repris dans Contes cruels, Séguier, II, pp. 604-605.

${ }^{15}$ Scrguci Pankejeff, plus connu dans l'histoire de la psychanalyse sous le qualificatif de «l'homme aux loups », en témoigne : "Quand je parlais à Freud de ma prédilection pour Maupassant, il répliqua : "Vous avez bon goût ». Comme l'écrivain français Mirbeau qui abordait des thèmes très
} 
Fort troublante est l'exemplarité pathologique du traumatisme qui marque la sensibilité du petit Georges Robin : choc psychique face à ce qui paraît une scène de violence, voire un crime ${ }^{16}$; modification de son attitude devant les parents, accompagnée de peur, de dégoût et de honte ${ }^{37}$; reconnaissance d'une énigme fascinante, qui sollicite de manière trouble la curiosité des enfants ${ }^{18}$.

La critique des sources a permis de notables avancées dans la connaissance, lacunaire jusqu'en 1991, de la personnalité de Mirbeau et de l'étendue des rapports de l'œuvre avec sa biographie. Un travail parfois nourri d'hypothèses, bâti sur des suppositions que seules l'intuition et l'empathie étayaient, reposant sur la conjecture plus que sur la certitude positive, s'avérait riche en frustrations, suscitait les polémiques, relançait le débat sur les risques d'une lecture «biographique» de l'œuvre romanesque, mais témoignait de l'intérêt réel à éclairer des zones d'ombre foisonnantes.

En définitive, il s'avérait correspondre si ce n'est à la lettre, au moins à l'esprit d'une rencontre entre une sensibilité et une époque. Savoir si le jeune pensionnaire du lycée Saint François-Xavier de Vannes a bel et bien subi les violences sexuelles d'un père jésuite semble maintenant devoir échapper à tout mode de vérification infaillible. Il apparaît aujourd'hui qu'il n'en est pas moins nécessaire d'envisager cette hypothèse comme une éventualité, susceptible de fonctionner, à l'égard de la création littéraire comme de la complexité relationnelle de Mirbeau au monde, à l'instar d'une clé. Des motifs d'une autre nature - comment prendre "positivement " la mesure de l'influence d'un proche sur un tempérament qu'elle modifie, altère ou pousse au contraire sur sa ligne de meilleure pente ? - rendent impossible le discemement du rôle exact de la rencontre avec Alice Regnault sur le psychisme de son époux. Il n'en est pas moins capital, conme l'ont fait Pierre Michel et JeanFrançois Nivet, de souligner avec netteté sa place, ne serait-ce que pour assumer le courage de considérer de face certains aspects moins "glorieux» de la vie de l'auteur de Sébastien Roch, tel le lâchage de Camille Pissarro ${ }^{19}$ ou la publication du testament patriotique dans Le Petit Parisien, qui suscite autant de questionnements que la possibilité du viol : il était dit que la vie de Mirbeau serait enchâssée entre

osés était alors à la mode, je demandais s'il lui plaisait; la réponse fut tout à fait négative " dans L'Homme aux loups, "Mes souvenirs sur Sigmund Freud», cité par C. Georges dans Octave Mirbeau, une porte, L'Âne, octobre 1993.

${ }^{16}$ « D'abord, j'ai cru qu'ils se baltaient, qu'ils s'égorgeaient... Le lit craquait... ma mère hurlait... la voix étouffée, la voix étranglée... Mais non une fois, j’ai vu... c'étaient des saletés !... », op. cit., p. 580 .

${ }^{17}$ " Je n'ose plus les regarder en face... Rien que de sentir passer la robe de ma mère, près de moi, je rougis... Car je ne les vois plus tels qu'ils sont lorsque je les vois... Je les vois toujours, comme la nuit... ", op. cit.

18 "Quant à moi, je ne pensais ni à l'oncle Jules, ni au cousin Debray [...] et mes yeux allaient de Mme Robin, qui me semblait moins laide, au lit d'acajou, au-dessus duquel un mystère planait, sous les draperies blanches ", op. cit., p. 582.

${ }^{19}$ Voir Octave Mirbeau, op. cit., pp. 484-485. 
deux points d'interrogation... Manifestement, nombre de zones d'ombre nous empêchent encore de saisir exactement en quoi la vie et particulièrement l'enfance de Mirbeau a pu contribuer à façonner ce caractère d'exception.

C'est pourquoi, sans poursuivre le dessein d'une explication clinique ou pathologique, il nous a semblé édifiant, en vue de nous familiariser avec un imaginaire torturé et portant des marques qui ont peut-être à voir avec un ou des événements réels et vécus dans l'enfance, de mettre en parallèle quelques-uns des traits qui ont composé le drame de ce patient que Freud suit de 1910 à 1914, et qui ne ressortit en rien du domaine artistique, et plusieurs des composantes du tempérament, des idées ou du parcours biographique de l'auteur des Vingt et un jours d'un neurasthénique. On connaît assez l'intrication du talent, a fortiori du génie, et de désordres psychologiques décrivant une gamme infinie de variété et de degrés de gravité. Ce rapprochement, non exempt de tâtonnements et pour spéculatif qu'il soit permettra peutêtre de préciser la nature et la profondeur d'un traumatisme - a-t-il été physiquement vécu par Mirbeau ? -, plus encore de prendre conscience du travail de transmutation littéraire d'un «matériau » qui, considéré exclusivement du point de vue du praticien, semble river celui qu'il affecte à un rôle de passivité et d'invalidité créatrice et relationnelle.

\section{MIRBEAU, UN HOMME AUX LOUPS ?}

Le fait est que mises à part la précision minutieuse des contours de la pathologie caractéristique de Sergueï Pankejeff ${ }^{20}$ et l'exploitation de ses talents dans le cadre d'une carrière artistique chez Mirbeau, le rapprochement de deux personnalités résolument placées sous le signe de la neurasthénie et de la toutepuissance du psychisme ne laisse pas de suggérer les partis à tirer d'une plus ample étude de la psyché et de l'enfance de l'écrivain.

Quoi de plus proche, en effet, de la configuration à la fois contradictoire et puissamment cohérente de la vie de Mirbeau, que la définition que Freud donne du parcours intérieur de son patient, chez qui persistent dans le temps des attitudes que, chez d'autres, les nécessités de la vie sociale enfouiraient sous un vernis opaque ?

Un remplacement instantané et sans encombres d'une phase par la suivante ne se trouve ni dans la nature des choses ni dans celle de notre patient, pour qui étaient au contraire caractéristiques le maintien de tout ce qui avait précédé et la coexistence des courants les plus divers ${ }^{21}$.

20 Encore que certains troubles chez Mirbeau, les crises d'insomnic notamment, qu'après recherches il identifiera sous le nom de "corybantisme", rendent regrettable pour le chercheur le fait que l'écrivain ne se soit jamais prêté au jeu du divan...

${ }^{21} \mathrm{~S}$. F reud, La Névrose de contrainte, CEuvres complètes, tome XIII, pp. 58-59. 
Force est de reconnaître que les phases déterminantes de la vie de Mirbeau peuvent être considérées comme très proches de certaines étapes condensées de l'âge tendre de "l'homme aux loups ». Nous le rappelons une dernière fois, il ne s'agit pas ici de faire de l'étude clinique d'un cas pathologique une extrapolation dont l'œuvre et la personnalité de Mirbeau seraient les objets, mais de montrer comment une certaine sensibilité profonde - peut-être conditionnée par des événements de l'enfance - travaille l'imaginaire créateur et incline l'auteur vers une symbolique dont lui-même ignore sans doute les tenants et les aboutissants.

Mentionnons ainsi quelques-uns des événements qui font sens dans l'enfance du patient de Freud, en précisant que ces attitudes sont celles d'un enfant, et que nous les rapprochons pour l'essentiel de celles de Mirbeau adulte. Néanmoins les quelques aperçus qu'autorisent sur la prime jeunesse de l'écrivain la lecture de l'œuvre et le témoignage de ses contemporains vont dans le même sens.

Ainsi l'attitude de révolte spontanée chez Mirbeau enfant : en témoignent aussi bien le Journal des Goncourt, évoquant « le petit rousseau que c'était, quand il était enfant, et le terrible affronteur ! ... Il avait, par bravade, la manie de se jeter sous les pieds des chevaux de $[\mathrm{m}]$ es voitures et de celles des d'Andlau ${ }^{22}$ - et ce rapport conflictuel de l'enfant, avec les grands animaux ${ }^{23}$ que Freud signale comme symptomatique - que la fiction : L'Abbé Jules évoque l'enfance indomptable du héros éponyme qui manifestement «veut provoquer la punition ${ }^{24}$. La révolte de l'écrivain d'âge mûr rappelle elle aussi celle du malade soigné par le professeur viennois. Chez l'écrivain qui perd sa mère à vingt-deux ans, en 1870, l'opposition au père ne semble pas avoir été prépondérante, même si la transposition romanesque, on le verra plus tard, permet des hypothèses d'un tout autre ordre. Relevons surtout le caractère variable du rapport au père, qui suscite les repentirs ${ }^{25}$ du fils au moment où paraît Le Calvaire, réquisitoire à peine voilé contre les décalages entre un père humain mais obtus et sa famille, mère et fils confondus, et dont la mort en 1900 semble avoir généré chez l'écrivain qui a passé la quarantaine une douleur sincère.

C'est ce même jeu de fascination / répulsion, moteur de la sensibilité de Mirbeau, qui constitue sans doute la pierre d'angle du rapprochement entre le romancier à la trajectoire en lignes brisées et le cas pathologique qu'étudie Freud. En effet, peut-être serait-il fructueux d'envisager les nombreuses hésitations du parcours de Mirbeau, perpétuellement déchiré entre adhésion totale et rejet violent en termes psychanalytiques de sublimation, effective, puis abandonnée. Ainsi de la fascination-répulsion pour l'argent, objet qui cristallise toul d'abord les désirs au temps de la liaison avec Judith Vimmer, pour qui Mirbeau dégagera de gros

${ }^{22}$ Journal, Goncourt, op. cit., II, p. 374.

${ }^{23}$ S. Freud, L'Homme aux loups, tome XIII, p. 23.

${ }^{24}$ S. Freud, op. cit., p. 25.

${ }^{25}$ Voir Octave Mirbeau, op. cit., p. 288. 
bénéfices boursiers, puis une sorte de dégoût symptomatique mentionné dans $L a$ $628-E 8$, où il convie notamment les peuples les moins «civilisés » à s'en éloigner en l'enfouissant de manière significative ${ }^{26}$.

Ainsi bien évidemment de l'attitude vis-à-vis de deux discours, le discours sur l'armée et l'attitude face au religieux. Tous deux suscitent dans le temps des réactions contrastées, qui se traduisent notamment par l'ambivalence des engagements idéologiques et politiques. Entre la sublimation religieuse spontanée du jeune Roch, semblable à son auteur dans le sens où sa religiosité n'est que le fruit d'une sensibilité exigeante, et l'athéisme violent de Mirbeau, entre son anticléricalisme et son besoin d'une certaine spiritualité, la gamme des postures face aux phénomènes de croyance ne reflète-t-elle pas profondément ce que Freud met sur le compte du rejet de la figure du père, successif ou simultané à un attachement filial d'une même intensité ? Nulle part ailleurs mieux que dans L'Abbé Jules, le deuxième roman, ne se lit la consubstantialité du désir et de la vocation mystique :

Dieu, ce n'est qu'une forme de la débauche d'amour! ... C'est la suprême jouissance inexorable, vers laquelle nous tendons tous nos désirs surmenés, et que nous n'atteignons jamais [...] Elle [mon âme] frémit, remuée en tous ses vagues enthousiasmes [...] comme ma chair frémit, secouće en toutes ses moelles devant une fermme nue, ou seulement devant son image rêvée... ${ }^{27}$

De même, chez Mirbeau, le rejet ou l'apologie de l'autorité, militaire cette fois, semble avoir une fonction de compensation. L'attitude quasi extatique de la foule et de Marguerite devant le passage d'une compagnie de dragons renseigne le jeune Sébastien Roch sur l'intrication de la notion d'hérö̈sme avec la séduction masculine dans ce qu'elle a de plus profond et d'instinctif ${ }^{28}$. Le transfert de l'image du militaire à celle du père est attesté par la même attitude versatile qui marque le rapport au père réel de Mirbeau. Il s'en faut de peu d'années pour que l'appel à la rigueur d'un dictateur, dans les colonnes des Grimaces ou l'éloge de la caste, dans les Lettres de l'Inde, voisinent dans le temps avec la profession de foi antimilitariste et anticléricale qui fait office de dédicace paradoxale au Jardin des supplices. Et n'est-ce pas le rejet inexorable du père qui se lit en filigrane de ces quelques lignes?

Aux Prêtres, aux Soldats, aux Juges,

aux Hommes,

qui éduquent, dirigent, gouvernent les hommes,

je dédie

ces pages de Meurtre et de Sang

Retenons quoi qu'il en soit le tremblement des attitudes, sur le terrain du religieux comme face à l'autorité militaire, ces deux avatars de la représentation du

\footnotetext{
${ }^{26}$ La 628-E 8, 10/18, 1977, p. 234.

${ }^{27}$ L'Abbé Jules, op. cit., p. 110.

${ }^{28}$ Sébastien Roch, pp. 1005-1007.
} 
père, chez cet anarchiste que Gustave Geffroy dépeignait comme ayant l'allure d'un «demi-solde batailleur de la Restauration»" Jules Renard comme «un type d'adjudant d'artillerie $"$ ". Ultime illustration de ce qui n'est peut-être qu'une forme de cette névrose de contrainte: le caractère systématique de son antisémitisme, auquel se substitue rapidement un philosémitisme aussi insolite - en soulignant en outre que, comme pour le patient de Freud, la signification de la circoncision équivaut à une castration atténuée ${ }^{31}$ : aussi bien l'écriture du polémiste développe-telle dans ses chroniques une rhétorique dénonçant volontiers les atteintes à la virilité morale, les entreprises politiques d' "émasculation », les effets «dévirilisants" de l'éducation ou de la "castration » qu'opèrent sur l'individu les pressions de la société. Sa relation problématique aux Juifs peut-elle d'ailleurs être considérée autrement qu'à la lumière de la psychanalyse, lorsqu'on prend conscience de la place du sentiment de culpabilité que Mirbeau développe très rapidement après le délire antisémite des Grimaces ? Après la violente hostilité, la tentative de rédemption auprès de ses anciennes cibles passe aussi par la voie littéraire, en témoigne le récit des pogroms dans $L a 628-E 8$.

En outre, à bien regarder le discours de Mirbeau, on pourrait trouver dans le déchaînement de certains de ses personnages contre un Dieu cruel, un contrepoint surprenant avec l'image valorisée du Christ ${ }^{32}$, bien souvent assimilé au premier des anarchistes, plein d'une ardeur révolutionnaire ; cette récupération ne serait pas si loin de celle qu'opère Barbusse, dans un sens communiste plus qu'anarchiste, dans Les Judas de Jésus, en 1927. Le Fils, préféré au Père, n'apparaît-il pas comme un indice probant de ce complexe d'CEdipe positif?

D'autres motifs appartenant à la vie personnelle de l'écrivain abondent en ce sens. Son attitude infantile à l'égard de sa femme Alice (il cache son mariage à ses meilleurs amis, se soumet aux caprices les plus lourds de conséquences sur son amitié avec Pissarro, par exemple, etc.), l'ambiguïté de son rapport aux enfants, particulièrement aux nouveau-nés, source de répugnance ${ }^{33}$, la nature mal connue de son rapport à ses sours - dont seul Dans le ciel dresse une esquisse romanesque, qui correspond à peu de choses près à l'attitude de méchanceté de la sœur du patient de Freud, aiguisant le sentiment de jalousie vis-à-vis des parents - permettent sans grands risques d'erreur d'imaginer la large place laissée à la figure maternelle dans l'inconscient de l'écrivain. Identification et rejet du père, amour et défense de la mère, toutes les contradictions et la dynamique à l'œuvre dans la sensibilité anarchiste semblent s'enraciner dans cet indissociable nœud du psychisme.

${ }^{29}$ G. Geffroy, Souvenirs de Mirbeau, Cahiers d'aujourd'hui, n ${ }^{\circ}$, 1922, p. 101.

${ }^{30}$ Joumal, Renard, Robert Laffont, Bouquins, 3 février 1891, p. 61.

${ }^{31} \mathrm{~S}$. Frcud, L'Homme aux loups, op. cit., p. 83.

${ }^{32}$ Voir la chronique Paul Gauguin, L'Écho de Paris, 16 février 1891, repris dans Combats esthétiques, I, p. 419, Les Mauvais Bergers, IV, 2, repris dans Théâtre complet, p. 119, La 628-E 8 «Les Juifs sont très préoccupés de Jésus... Weil-Sée aimait à en parler [...] $\wedge u$ fond, il était fier d'avoir un Dieu dans sa famille », p. 250 ou Dingo, Fasquclle, 1913, p. 292.

${ }^{33}$ Dingo, op. cit., p. 30 . 
À l'évidence, la nécessité d'un rapprochement de la nature sous une forme à laquelle l'imaginaire de Mirbeau doit beaucoup, le mythe, tisse des liens profonds avec le visage fantasmatique de la mère, menacée, bafouée, de qui l'écrivain se sent appelé à prendre la défense, mais qui en échange dispense un sentiment de sérénité. L'identification à la mère autorise surtout Mirbeau à faire sien l'état de créateur. La référence à Isis relève avant tout d'une rêverie sur la possibilité de créer.

\section{LES RAPPORTS PĖRE ET FILS : UN CONFLIT FREUDIEN ? LE PĖRE COUPABLE}

Maternelle autant que matricielle, l'écriture assoit ses fondements profonds en se faisant, de surcroît, parricide. Père monstrueux, vicieux, ou père réduit au silence et à la passivité, on retiendra de la figure paternelle sa négation par l'espace romanesque.

Le déni d'existence se lit selon ces deux modalités, par lesquelles le texte prend, en quelque sorte, la défense de la mère brimée.

\section{LE PĖRE MONSTRUEUX}

La crise de folie du père Roch menaçant la vie de son fils ${ }^{34}$ est une incarnation par l'allégorie de ces meurtres virtuels de personnalités enfantines, commis tout au long de l'œuvre par des parents soucieux de reconnaissance sociale ou cherchant à prendre leur revanche sur une vie insignifiante. Les parents du narrateur du Jardin des supplices écrasent leur rejeton sous le martèlement de leur cupidité ; le couple Dervielle bride les désirs de liberté et la curiosité du fils ; les parents du narrateur de Dans le ciel déterminent le caractère dépressif de leur enfant : tous cuvrent à l'infanticide moral ; leur comportement traduit chez le narrateur un profond sentiment de la médiocrité parentale face à la spontanéité créatrice ou au désir impérieux de connaître et d'apprendre manifestés par l'enfant. Peu de velléités de riposte, encore moins de parricide, viennent répondre à ces brimades. L'opposition courageuse de Sébastien au geste de meurtre de son père reste l'expression physique la plus significative, à quoi il convient d'ajouter, dans Les Mauvais Bergers, le sentiment qu'éprouve Hargand d'être sacrifié par son fils sur l'autel de l'idéologie ${ }^{35}$.

\section{LE PĖRE ABSENT}

Encore faut-il prendre en compte la possibilité pour le fils de concevoir un amour sincère pour son père, une compassion filiale ardente vis-à-vis de défauts paternels dont bien souvent il est pourtant la première victime. Jean mesure avec le

${ }^{34}$ Fin du livre I, chapitre VIJ.

${ }^{35}$ Acte III, scène 2, repris dans Théâtre complet, Éditions InterUniversitaires, Mont-de-Marsan, 1999, p. 94. 
recul du temps que son tempérament n'est pas si éloigné des faiblesses du père Mintié. Le fossé affectif qui s'est creusé entre Sébastien et un père lui aussi veuf s'avère susceptible d'être comblé, à la faveur d'une prise de conscience de l'amère douleur qui imprègne toute chose humaine. Les deux tuteurs partagent somme toute le triste privilège de la nécessité d'élever seul un fils qui leur en sait gré sur le tard.

Il revient néanmoins à certains des fils de dépasser l'insipidité du caractère paternel, en tentant de dispenser une consolation qui échoit traditionnellement aux liens maternels. Le Calvaire offre, on l'a vu, une illustration insolite de cet altruisme, dans un cadre a priori incompatible avec ces élans : au plus fort de la débâcle de 1870, Mintié est gagné par un sentiment de charité et de solidarité à l'égard de ces pauvres diables engagés dans la spirale des conflits meurtriers, et s'imagine sous les traits d'Isis, alimentant de ses cent mamelles ces orphelins douloureux. Ici, la présence de la Mère ne se décline pas sur le mode féminin. L'armée, l'institution scolaire ou les fréquentations artistiques servent ainsi bon gré mal gré de creuset où s'éprouve la réalité du lien entre hommes, qui chez Mirbeau suscite la nécessité significative d'une réintégration fusionnelle à la mère.

\section{UNE DIMENSION INCESTUEUSE}

Fait significatif, l'impossible réversibilité des situations filiale et paternelle se colore d'une nuance psychanalytique quand elle met en situation de rivalité père et fils, soudés par une même attirance pour la figure de la mère. On l'a vu, la trame narrative inscrit en filigrane, çà et là, les motifs d'un partage entre hommes qui tient de l'inceste. Ainsi, l'allusion à la transgression est-elle plus éloquente dans la scène où Sébastien Roch, l'esprit prompt à attribuer à sa mère défunte une expérience adultère, s'empare des vieux vêtements maternels, et « les embrasse, presque furieusement, d'un long, d'un horrible, d'un incestueux baiser ${ }^{36}$, ou dans ses tentatives de séduction dirigées vers la mère de Marguerite ${ }^{37}$. De manière moins explicite, le fantasme de l'acte interdit se lit dans les nombreuses évocations de la présence de la mère au moment de l'étreinte. Il va de pair avec l'affaiblissement moral causé par la femme tentatrice, et s'inscrit dans les innombrables formes du sentiment de culpabilité : après la sauvage étreinte qui les a unis furtivement, Marguerite, près de Sébastien effondré, croit "jouer à la maman avec sa poupée »" La jeune Julia, dans Dans le ciel, se pâme dans les bras inexpérimentés du narrateur en balbutiant "Maman !...Maman !... »". On ne s'est peut-être pas assez avisé que ce que l'on a qualifié, à la suite de Léon Daudet, de "gynécophobie », esquisse souvent chez

\footnotetext{
${ }^{36}$ Op. cit., p. 992.

${ }^{37}$ Ibidem, pp. 1015-1016.

${ }^{38}$ Ibidem, p. 1050.

${ }^{39}$ Dans le ciel, p. 115.
} 
Mirbeau une situation incestueuse, auprès d'une femme dont le visage se modèle sur les contours de celui de la mère.

En définitive, et pour en revenir au rapprochement qui a impulsé notre propos, il s'avère que «la peur du loup », et son corollaire, le souhait de protection de la mère, constituent un moteur fondamental de l'écriture, chez Mirbeau. L'acte de créer s'enracine ainsi en cette absence qu'il faut combler. Il se trouve conditionné par le modèle matemel sous-jacent. Figure au sens métaphorique et dans l'acception psychanalytique du terme, la mère, à la fois désirée et victime, absente et surdéterminée, fantasmée et réelle, est bien à l'origine de cette hypostase première qu'est la nature, pour Mirbeau. Isis aux traits complexes, elle se place au principe de son écriture, en figurant aussi bien les signes d'un malaise, que la possibilité d'une compensation.

\section{POUR UNE SYNTHĖSE.}

\section{LES FORMES D'UNE DISSIDENCE : LES DEUX FONCTIONS DE LA NATURE}

La complexité de l'objet idéal de nature rend plus évident le phénomène d'introjection dont elle fait l'objet chez Mirbeau, qui viserait à conforter un sentiment de sécurité et d'équilibre. L'intuition d'une nature doublement nécessaire - elle apporte enrichissement intellectuel, stimule l'ouverture sur un monde varié et ses mécanismes, en même temps qu'elle appelle à un dépassement d'ordre spirituel vers une compréhension plus vaste de la place de l'homme - relève en effet pleinement du mythe, dans le sens où s'exprime à travers elle une somme d'attentes profondes et fondamentalement contradictoires. Il est étonnant en effet de constater comment chez Mirbeau l'intérêt marqué pour la science ne nuit pas à une vision anthropomorphiste de la nature, comment la vivacité de sa croyance dans le progrès scientifique n'est nullement altérée par la revendication d'une compréhension supérieure, plus spéculative, qui éclairerait le rôle et la place de l'humain : il s'agit de « voir, sentir », mais aussi de " comprendre ${ }^{40}$.

Il semble que Mirbeau redouble là de prudence et se paie le luxe de deux stratégies de protection, si l'on considère ainsi, à la suite de Freud, le rôle à jouer par ces attitudes mentales. Dans un monde « où les Dieux ne sont plus maîtres » selon Freud, à plus forte raison chez un auteur qui s'enorgueillit de participer à leur fuite, la science ne contente pas au point de libérer de jeux psychologiques qui prennent pour objet la nature. Dans cette tactique de sécurisation maximale, qui n'élit pas l'une plutôt que l'autre tendance, toute l'âme enfantine, naïve, composée, de Mirbeau se laisse entrevoir. Âme enfantine ou «pensée sauvage " pour reprendre le titre de Claude Lévi-Strauss, pour qui « loin d'être, comme on l'a souvent prétendu,

\footnotetext{
${ }^{40}$ Ibidem, p. 87.
} 
l'œuvre d'une "fonction fabulatrice" tournant le dos à la réalité, les mythes et les rites offrent pour valeur principale de préserver jusqu'à notre époque, sous une forme résiduelle, des modes d'observation et de réflexion qui furent (et demeurent sans doute) exactement adaptés à des découvertes d'un certain type : celles qu'autorisait la nature, à partir de l'organisation et de l'exploitation spéculaire du monde sensible en termes de sensible ${ }^{41}$.

\section{RÔLE DE MATURATION ET D'AFFRANCHISSEMENT SPIRITUEL}

Le rôle de compensation que les personnages attendent de la nature tient à l'apport de beauté et résulte de la satisfaction d'une demande d'harmonie, d'équilibre : cette beauté s'inscrit dans la perspective d'une quête d'absolu, et prend sens quand on considère que seul le soulagement qu'elle procure doue de signification et de légitimité la place de l'être ici-bas. À l'infini de l'attente des personnages, répond l'infini des richesses que la nature dispense à l'homme. X., l'un des narrateurs de Dans le ciel, déplore que son jeune âge ne lui permette de connaitre «le sentiment, si rassurant, si égoïste, de la beauté éparse dans les choses, de la beauté qui, seule, suffit à expliquer, à excuser ce malentendu, ce crime : l'univers ${ }^{42}$.

Cet ordre parfait et cette beauté du monde relèvent d'une vraie transcendance et laissent fugitivement entrevoir aux sensibilités d'artistes et d'enfants (bien souvent les deux à la fois) l'horizon que peu d'entre eux parviennent à atteindre ; il s'agit d'un même coup de satisfaire une quête d'ordre esthétique et de parvenir à un état de sevrage affectif. Le même personnage se désole de ne pas trouver « dans la prunelle des hommes, au calice des fleurs, aux formes si changeantes, si multiples de la vie » quelque chose « qui correspondit au vague et obscur et angoissant besoin d'aimer qui emplissait [s]on cœur, gonflait [s]es veines, tendait toute [s] a chair et [s]on âme vers d'inétreignables étreintes, vers d'impossibles caresses »".3. Comment définir l'objet de l'attente, dès lors que n'est pas identifiée la nature de ce besoin même ? C'est cette interrogation problématique qui nourrit les angoisses de personnages que leur sensibilité d'écorché vif condamne à une incertitude permanente. En définitive, ces questionnements laissent davantage apparaître un espace de manque, une béance chez ces individus, plus qu'ils ne renseignent sur les modalités de sa satisfaction. Avant d'être en quête de savoir, ils sont indéniablement orphelins d'une présence sécurisante, et projettent sur la nature et sa beauté consolante l'angoisse vertigineuse que suscite cet inconnu. La fin du XIX ${ }^{\text {ème }}$ siècle dans laquelle s'inscrivent les premières ceuvres de Mirbeau porte le deuil de Dieu, des dieux, qui ont pu précédemment combler de telles aspirations à l'absolu.

\footnotetext{
${ }^{41}$ Cité par P. Brunel, dans Le Mythe de la métamorphose, A. Colin, 1994, p. 95.

${ }^{42}$ Dans le ciel, op. cit., p. 47.

${ }^{43}$ lbidem.
} 
Avant de concevoir un substitut propre à endosser ce rôle, sous la forme du progrès scientifique dont l'engouement suscitera une certaine sorte de conversion, ou sous la forme de l'engagement politique illustré par les intellectuels que révèle l'affaire Dreyfus, ou sous la forme de la vocation artistique, la place autrefois occupée par la figure de Dieu reste vacante, et le genre romanesque se fait l'écho de cette carence chez des personnages dont la recherche spirituelle, soudain privée de support, semble toumer à vide (ainsi des premiers romans de Huysmans, des personnages tourmentés de Dostoïevski, ou de ceux des premiers drames de Claudel). Ce sentiment d'incomplétude est d'autant plus marqué chez Mirbeau que son évolution personnelle se superpose globalement aux mutations d'une pensée humaine qui vise, dans la seconde moitié du siècle, à l'affranchissement par la libération des croyances religieuses. Du respect de l'autel à la négation de la vérité religieuse, la représentation divine se voit destituée de sa légitimité par l'auteur de L'Abbé Jules. Mais qu'en est-il désormais de cet "angoissant besoin d'aimer » qui agitait l'âme des personnages? Comment s'épanche maintenant la soif de ce sens et de cette signification qui devraient orienter leur existence?

Seule la nature peut servir de réceptacle mythique, de point d'ancrage idéel à ces interrogations en suspens. C'est ainsi qu'il faut comprendre l'attachement que lui témoignent les jeunes protagonistes en mal d'affection et de connaissances. Il en résulte parfois un discours baroque, de la part d'enfants ou de rêveurs qui projettent sur elle des désirs divergents : ainsi le trouble et l'inquiétude de ne rien savoir se traduisent en termes qui dénotent à la fois une volonté d'approcher le sens d'une sorte de lieu sacré - les dénominations de « secret », $d$ " « énigme », de « mystère » transcrivent une distance craintive et apeurée - et le souhait plus prosaïque d'éclairer les mécanismes de la vie animale et végétale. Le personnage évoqué plus haut avoue partager les craintes du monde «affolé[...] par l'impénétrable énigme de la matière ou de la divinité ${ }^{44}$, mais n'a de cesse de chercher, devant une fleur par exemple, «le secret, le mystère de sa vie $»^{45}$. Le mouvement de translation de l'environnement biologique connu et familier des insectes et des fleurs, à la sphère du moi réflexif de l'enfant constitue d'ailleurs dans cet ouvrage l'occasion d'un va-et-vient récurrent entre microcosme physique et monde de l'intelligible; de l'araignée qui inculque à son hôte humain la loi fatale du meurtre ${ }^{46}$, à «la diatomée perdue dans l'eau vaseuse des citernes ${ }^{47}$ qui symbolise le sentiment de vacuité de son existence, en passant par les fourmis et les abeilles longtemps observées ${ }^{48}$ et l'image pascalienne de l'humain comme atome microscopique ${ }^{49}$, l'ordre animal conçu sous sa forme la plus humble, loin de ne représenter que l'objet d'une

\footnotetext{
${ }^{44}$ Ibidem, p. 56.

${ }^{45}$ Ibidem, p. 58.

${ }^{46} \mathrm{Ibidem}$, p. 67.

${ }^{47}$ Jbidem, p. 56.

${ }^{48}$ Ibidem, p. 58.

${ }^{49}$ Ibiden, pp. 47, 48, 55 .
} 
curiosité scientifique, est le résumé paradoxal d'une interrogation métaphysique sur la condition de l'homme, ce "silencieux insecte ${ }^{50}$. Mirbeau trouve sans conteste dans ce partage de l'infiniment petit et de l'infiniment grand une communauté d'intuitions avec le poète Maeterlinck dont il a révélé au public La Princesse Maleine quelques années auparavant, même si son symbolisme animal ne se départit pas de la dimension biologique.

Pour autant, la recherche d'une intelligibilité de la destinée humaine à travers l'observation des phénomènes naturels ne s'enferme pas dans la contemplation du monde microscopique : le Père Pamphile, autre enfant parvenu à l'âge adulte sans avoir rien abdiqué de sa capacité de rêver, parvient à un tel point d'obsession qu'il en vient à confondre mentalement l'image de ses projets, la chapelle du Réno, et le cadre naturel. La vision animiste est ici grandiose, démesurée, mais traduit la même plasticité d'une nature protéiforme vers laquelle se transfère l'idéal mystique et visionnaire du prêtre :

Et la chapelle emplissait la terre, emplissait le ciel. Le ciel était sa voûte, les montagnes étaient ses autels, les forêts ses colonnes, l'Océan ses baptistères, le soleil son ostensoir, et le vent ses orgues ${ }^{51}$.

\section{DIALECTIQUE DE LA SCIENCE ET DU SPIRITUEL : UNE QUESTION D'ÉPOQUE}

Quelque neuve et complète que puisse paraître la double perspective adoptée par Mirbeau, elle ne s'inscrit pas moins dans l'évolution d'une époque animée de la volonté de s'affranchir des dogmes religieux pour accéder à la lumière du savoir rationnel, mais encore loin du sevrage spirituel. L'homme du XIX ${ }^{\text {eme }}$ siècle remet en question le bien-fondé du discours religieux, sans que soit abolie sa capacité de questionner le sens fondamental des choses et de sa destinée. Les vérités de la science ne tiennent pas lieu de certitudes compensatoires au point d'offrir une assise morale; on ne tarde pas à prendre conscience que les postulats scientifiques ne sont guère plus sécurisants que les spéculations sur le sacré, et la loi de l'entropie suscite le trouble dans les mentalités. Le positivisme produit un discours dogmatique et figé. La philosophie du doute ne se fait pas scrupule de se retourner contre ce qui l'a dans un premier temps aiguillonnée. Le père fondateur du positivisme, Auguste Comte lui-même, ne s'emploiera-t-il pas sur le tard, à réévaluer grandement la place de la subjectivité dans toute analyse des phénomènes humains ? C'est cependant la silhouette de Renan qui s'inscrit en filigrane des tâtonnements de Mirbeau, Renan dont la sensibilité féconde et l'esprit critique ne parviennent à un équilibre que dans l'abandon à la toute-puissance du doute universel.

\footnotetext{
${ }^{50}$ Ibidem, p. 55.

${ }^{51} L$ 'Abbé Jules, p. 464.
} 
Mirbeau ne se contente pas de cette forme atypique de confort moral, et préfère résolument l'action. Cette dynamique, qui fait défaut à la pensée renanienne, incite Mirbeau à faire retour sur des questions auxquelles il lui semble que la pensée scientiste n'a pas su répondre de manière satisfaisante, voire qu'elle a éludées purement et simplement. Ce mouvement de repentir, qui vise à résoudre même $a$ posteriori les difficultés, à pallier les insuffisances et les lacunes dont s'accommode l'esprit positiviste, confère au questionnement une allure de va-et-vient perpétuel plus accentué encore que chez l'auteur de L'Avenir de la science (1848). Ainsi en 1907 on l'a vu, la modemité des inquiétudes écologiques n'abandonne-t-elle pas la nécessité de mieux se connaître moralement et spirituellement. C'est dire la plénitude nourricière et le contact fructueux que l'homme retire à plusieurs niveaux de son environnement ; c'est dire aussi la persistance et la vivacité chez Mirbeau du sentiment élémentaire, primitif, d'une nature perçue à la fois comme objet d'investigations scientifiques susceptibles d'affranchir l'homme de son ignorance, et comme espace infini du mystère qui l'entoure et le ramène perpétuellement à l'énigme de son moi et de sa place dans l'univers.

Cette complétude confine parfois à l'amalgame baroque, à une tension que seule la pleine liberté de l'artiste doue de sens : Jules Renard témoigne dans son Journal des angoisses qui assaillent les dernières années de Mirbeau, travaillé par la crainte de la mort et miné par l'altération de sa santé. Plus que jamais, l'apparition de ces grandes peurs active le processus d'enchevêtrement des deux attitudes face aux mystères de la nature, l'une puisant aux sources du rationnel, l'autre sollicitant le recours aux images traditionnelles du sacré :

22 janvier (1910) - Mirbeau me dit :

- Le corps est trop étroit. Dieu a mal fait ça. Des cailloux doivent y passer dans un vaisseau gros comme un cheveu. Et le cœur ! Est-ce mal fichu, le cœur $!^{52}$

Simple rhétorique ? La perspicacité de Renard lui permet de toucher du doigt le lieu où s'enracine le complexe du double profit à tirer de la nature, selon son ami de l'Académie Goncourt : à elle seule, l'imagination de "ce grand gosse de Mirbeau " ${ }^{53}$ fait fonctionner l'interpénétration active du corps - à éclairer de manière scientifique - et de l'esprit - dont la création artistique, le rapport à la nature doivent élucider le secret effrayant et tentant, "l'énigme de la matière ou de la divinité ${ }^{54}$. Jusqu'à une date avancée dans le siècle, l'incommensurable mystère de l'organisme physiologique et de l'organisation spirituelle voue l'homme à une peur qui fait ressurgir d'archaïques réflexes tissés de croyances et de superstitions. Mirbeau vieillissant se saisit à nouveau du moule de la doxa religieuse qu'a connu le jeune Octave durant les années passées sous la férule jésuite ; l'ardeur de son imagination est intacte, mais le support à investir suscite hésitations et confusions, et aboutit à

\footnotetext{
${ }^{52}$ Journal, 22 janvier 1910, op. cit., p. 994.

${ }^{53}$ lbidem, 28 janvier 1904, p. 697.

${ }^{54}$ Dans le ciel, p. 56.
} 
un discours inintelligible si l'on ne prend pas en compte cette part d'enfance intacte, inentamée, toujours active, chez celui qui faisait à Renard, toujours, "l'effet d'un timide enfant, d'un enfant terrible avec un cœur de mouton $»^{55}$.

En définitive, chez Octave Mirbeau, la nature est partagée entre deux fonctions : d'une part la fonction didactique d'une nature "expérimentale » qui donne lieu à une réflexion scientifique et appelle à l'affranchissement intellectuel ; d'autre part la fonction de guide spirituel d'une nature transcendante qui offre le seul espace où l'homme côtoie l'absolu : l'art lui-même, issu de ses beautés et de son travail, lui est inféodé. Cette division ne provient pas d'une démarche intellectuelle de Mirbeau. Bien au contraire, l'aspect irréductible des deux visées révèle l'enjeu vital, essentiel pour lui, d'une conciliation entre le Moi originel et le Moi social, l'adulte, engagés dans un perpétuel conflit.

Sans cesse, Mirbeau doit s'appliquer à plier le travail du génie aux exigences de la vie sociale tout en veillant à ne pas étouffer les forces vives de la création, entées sur le socle de la sensibilité infantile. Sans cesse, l'écrivain doit s'employer à ne pas laisser submerger son goût de la clarté et de la raison raisonnante par les débordements du fantasme et de l'imagination tyrannique.

De ces exigences découlent la conception d'un usage personnel du travail de création, une sorte « d'hygiène » de vie fondée sur la littérature : au même titre que chez des artistes qu'une semblable hantise habite et rapproche de Mirbeau - Van Gogh, Rodin, Claudel ou Mallarmé, le travail de l'art est vital, au sens où l'artiste se trouve «travaillé » par une mémoire lointaine, dont il ne finit plus d'accoucher.

Ce faisant, arrachant de soi la part odieuse, trop vive, Mirbeau prend conscience qu'elle est aussi le moteur vivant de la création. Il profite de cette faculté empoisonnée qui, selon Henri Michaux, désigne le génie : il s'agit de demeurer en contact avec "la zone vicieuse » de l'enfance. La conversion de ce matériau vénéneux, empoisonné, relève de la transmutation ; les contemporains de Mirbeau ont beaucoup glosé sur son travail re-créateur qui ne reposait que sur des facultés de déformation de ce qu'il observait. Roland Dorgelès parle d' « une machine à transfigurer le réel $»^{56}$. La principale opération de falsification est peut-être celle qui se nourrit, en s'en délestant provisoirement, du poids accablant des traumatismes et des manques qui débordent l'écrivain. Donner forme à l'informe et au chaos, tel qu'il a été vécu dès l'enfance ; Mirbeau constitue alors en source de la création son drame intérieur, son théâtre intime, il les insère dans un processus artistique d'où renaît perpétuellement un dynamisme - la vie.

55 Journal, 24 mai 1909, p. 980. Thadée Natanson parle de «son sourire où se nuançait une gentillesse d'enfant», Léon Werth évoque sa " voix d'enfant qui doute ", respectivement dans Sur des traits d'Octave Mirbeau, et Le pessimisme d'Octave Mirbeau, tous deux dans Les Cahiers d'Aujourd'hui, $n^{\circ} 9,1922$.

${ }^{36}$ Préface aux CEuvres complètes (1934), repris dans Euvres romanesques, Buchet-Chastel, 2000, p. 11 . 
Néanmoins le refus de construire dont témoigne chacune de ses œuvres, l'ensemble chaotique et disparate de l'œuvre qu'elles composent, montrent les limites de cet art. Ce mépris spontané de l'arrangement cadrant en définitive parfaitement avec l'exigence naturaliste de restituer le désordre apparent qui régit événements et phénomènes, ne serait-il pas le principal point d'attache de Mirbeau à la doctrine de Zola?

Le danger et la folie sont donc désamorcés par la discipline et l'ordre inhérents à l'œuvre d'art, et l'impuissance est compensée par le mouvement. Jules Renard toujours, s'interrogeait sur le contraste entre l'apparence et son cuvre : "Cet homme, de sens clair quand il écrit, a toujours en parlant quelque chose de fou ${ }^{57}$. La communication avec le monde ne peut s'opérer qu'au prix d'une réconciliation préalable avec soi, laquelle passe par l'écriture ; un dialogue fécond est rétabli, que permet la composition littćraire, lieu exclusif de la maturation de Mirbeau. Il s'agit, comme incline à le penser Marc Eigeldinger, "de reconquérir l'harmonie entre l'être et le langage qui a été indûment rompue " $"$.

La présence du mythe au cœur du texte manifeste toujours une réflexion vivante sur la parole. Réalité avant tout verbale, le mythe dit la persistance d'une interrogation sur le langage et ses limites. Il est nécessité de parler et pousse l'anarchiste à la formulation, lors même que celui-ci est convaincu du bien-fondé d'une économie de l'expression. «Voir, sentir, comprendre, tout est là » : après cette apologie de la pensée sans langage, est-il paradoxal de trouver chez cet écrivain, le mythe, qui est échange et parole ? C'est que le mythe réduit autant qu'il révèle cette tentation du silence ${ }^{59}$. Silence qui est avant tout une quête de consensus avec l'effroi de la nature monstrueuse ( "Taisez-vous », somme Clara au cour du jardin des supplices) ou avec les aberrations du désordre de la vie sociale. Silence de l'œuvre d'art, qui dans le strict respect de la pensée de Schopenhauer, invite à celui du spectateur. Silence de la littérature dont menace à tout instant Mirbeau, trop convaincu de la supériorité en éloquence vraie des arts plastiques.

Au vrai, la libération de cette parole inarticulée, primitive, ne pourra avoir lieu qu'après une sensible maturation du mythe, qui détermine réciproquement une métamorphose de l'écrivain. Ce terme de métamorphose ne convient-il pas parfaitement à cet auteur qui, débutant approximativement sa carrière d'homme de lettres alors qu'il est encore secrétaire de cabinet du préfet de l'Ariège, rencontre tous les succès en tant que romancier, passe avec un égal bonheur d'une inspiration autobiographique à un roman de critique sociale, via l'écriture d'un roman symboliste, pour au final s'abandonner derechef à sa tendance au récit personnel, et épouse enfin avec la fortune que l'on sait la carrière d'auteur de théâtre, menant encore de front un cohérent mais évolutif cheminement de critique d'art? Au discours des

\footnotetext{
${ }^{57} 12$ novembre 1907, loc. cit., p. 899 (daté par erreur du 12 septembre).

${ }^{58} \mathrm{M}$. Eigeldinger, Lumières du mythe, Presses universitaircs de France, p. 6.

${ }^{54}$ Voir notre élude : La loi du silence selon Mirbeau, Cahiers Mirbeau. ${ }^{\circ}$ 5, 1998, pp. 32-57.
} 
multiples perplexités sur une réalité révoltante et inassimilable, le mythe permet de substituer une représentation passionnée, mensongère parfois, vengeresse de cette réalité - et de s'en délivrer. Par cette mise à distance, par cette délivrance, Mirbeau, au tournant du siècle, peut à nouveau croire à ce superflu par lequel, selon l'un de ses personnages ${ }^{60}$, nous vivons réellement.

Conquérir un superflu nécessaire, ce luxe, cette Toison d'or: la route de Mirbeau est tout orientée vers le mythe.

\section{BIBLIOGRAPHJE}

Herzfeld C. (2001), Le Monde imaginaire de Mirbeau, Société Octave Mirbeau, Presses de l'Université d'Angers.

Lair S. (2004), Le Mythe de la nature dans l'auvre de Mirbeau, Presses Universitaires de Rennes. Michel P., Nivet J.-F. (1991), Octave Mirbeau, l'imprécateur au cour fidèle, Séguier.

${ }^{60}$ Sébastien Roch, p. 975. 